\title{
Allergy diagnosis: pros and cons of different tests, indications and
}

\section{limitations}

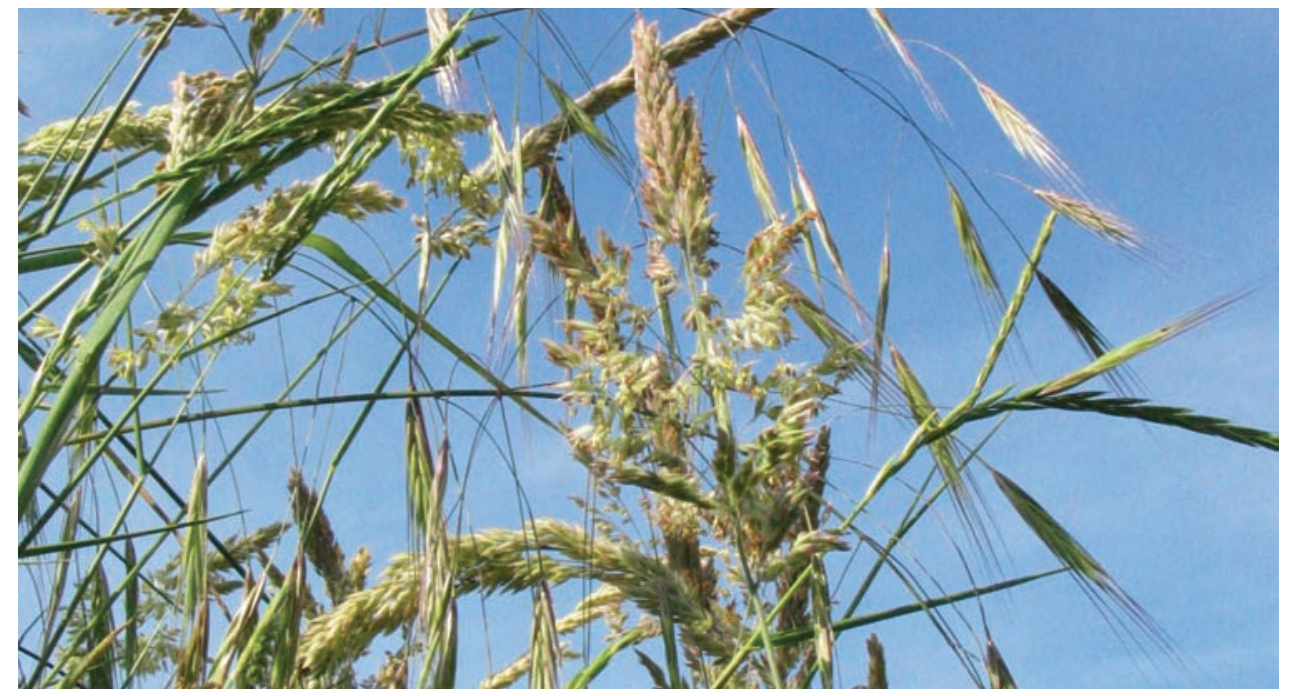

\section{Educational aims}

1 To describe different methods of allergy diagnosis, with pros and cons.

1 To explain the difference between sensitisation and allergy.

1 To demonstrate that allergic sensitisation is not an all-or-nothing phenomenon, but that the degree of sensitisation is also important.

\section{Summary}

Although related, allergy and sensitisation are not identical. It is quite common for sensitisation to occur asymptomatically, while "allergic" symptoms may also appear independent of the presence of sensitisation. In addition, the risk of allergic disease (symptoms) depends on the degree of sensitisation, rather than whether sensitisation is present.

The results of tests for sensitisation, such as skin prick tests (SPTs) and specific immunoglobulin (Ig)E tests, must be interpreted with this in mind. Because of their low cost and rapid results, SPTs are the preferred method to screen for allergic sensitisation. Specific lgE tests are more useful to confirm or reject the suspicion of specific sensitisation to a certain allergen. Caution is necessary when interpreting published results from tertiary-care reference centres; because the population studied is usually distinctly different from the patients in your centre, the results of such studies may not be applicable in your own clinical practice.
P.L.P. Brand

Princess Amalia Children's Clinic
Isala Klinieken
P.0.Box 10400
8000 GK Zwolle
the Netherlands
Fax: 31384247660
E-mail:p.l.p.brand@isala.nl

\section{Key points}

\Allergy and sensitisation are not the same.

I Sensitisation to both food and airborne allergens may change over time.

I Double-blind placebocontrolled food challenges are by far the best way to diagnose food allergy.

\ Size matters: the degree, not the presence, of sensitisation determines risk of allergy. 
Paediatric chest physicians think about allergy as a causative factor for respiratory symptoms each and every day. Most doctors have a routine approach to allergy testing, according to their personal preferences, hospital policy or both. Tests usually include SPTs (figure 1) or specific lgE tests, with a routine batch of allergens being tested. Since food allergy is not often considered as a cause of respiratory symptoms, the routine batch usually includes only inhalant allergens. Only in young children are food allergens included in screening for allergy.

Each method of allergy testing has its own pros and cons, indications and limitations. It is important to realise that there is an important difference between sensitisation (the presence of specific $\lg$ E antibodies, as indicated by a positive IgE test or SPT) and allergy (including symptoms caused by exposure to the allergen). In order for symptoms of allergy to develop, not only is sensitisation a prerequisite, but there also needs to be exposure to the allergen and a certain degree of end-organ hyperresponsiveness.

Figure 1.

Skin-prick test.
Only when these three conditions are met will atopic disease become discernible. This has important implications for allergy testing. On the one hand, there will be sensitised individuals who do not develop symptoms because they are not (sufficiently) exposed to the allergen under study, or because they fail to exhibit end-organ responsiveness. These individuals will be sensitised asymptomatically. On the other hand, studies have shown that quite a significant number of individuals with atopic disease, such as asthma, eczema or rhinitis, do not show sensitisation to specific allergens. It appears, therefore, that routine allergy testing can yield both false-positive and false-negative results. Most importantly, it is imperative that physicians realise that a positive allergy test does not prove allergy - it merely shows sensitisation. As a result, the term allergy test should be avoided and it should be replaced with the term sensitisation test.

\section{SPTs}

The SPT is the most commonly applied procedure for the investigation of possible sensitisation to allergens. The SPT is more popular in the USA than in (continental) Europe, but it is unclear why this is so. SPTs are easy to perform, and yield rapid and visible results, which are easily demonstrated to the patient and parents. Contraindications include the use of antihistamines (which reduce skin reactivity) and severe eczema. There is also a minute, but not absent, risk of serious side-effects (anaphylaxis). The use of well-standardised allergen extracts is advised. Although the prick-to-prick test may be used to assess skin reactivity to fresh foods, the relevance of these foods in determining symptoms can only be reliably examined by (doubleblind, placebo-controlled) food challenges.

SPTs are cheap (approximately $€ 20$ for materials for a full-batch screening test). Allergens can be stored in the refrigerator for about 1 year. Contrary to popular belief, SPTs can also be performed in young children, and both population and clinical studies have shown responses to both inhalant and food allergens in infants and toddlers. However, SPTs can be distressing for young children, and are best used in children $\geq 4$ years of age.

Based on these characteristics, skin prick testing is the ideal method of screening for atopic sensitisation in children aged $\geq 4$ years, unless they are using antihistamines or have serious atopic eczema. 


\section{Specific IgE measurement}

Specific IgE can be measured reliably and reproducibly in the serum of children, regardless of their age. Although, for clinical purposes, venipuncture is usually performed to obtain a blood sample for specific lgE testing, it is technically possible to perform specific lgE measurements in capillary blood samples obtained on filter paper. This method is most commonly used in research settings.

The measurement of total IgE serves no purpose in allergy diagnosis. The large degree of overlap in total IgE levels between healthy individuals and patients with atopic disease precludes any useful application in practice. Moreover, specific IgE to relevant allergens can be found in the presence of perfectly "normal" total IgE values. Conversely, high total IgE levels may be found in the absence of specific lgE sensitisation.

The main advantages of specific lgE measurements over the SPT are that virtually all allergens can be tested, and the results are not influenced by the use of antihistamines or eczema. In addition, there is no risk of side-effects. The disadvantages of specific IgE measurements include the relatively long time taken to produce results (usually 1-2 days), the burden of venipuncture and the relatively high cost (about $€ 20)$ per measurement. Based on these characteristics, specific lgE testing is best suited for the confirmation or refutation of the suspicion of clinically relevant specific sensitisation(s) to one or more allergens. This can be done in two ways: either by targeted measurements of specific allergens, or by the performance of a screening test, which is only determined further if positive.

It is well known that sensitisation to food allergens tends to diminish over time. This has been demonstrated both for SPTs and for specific lgE measurements. It is much less well known that sensitisation to aeroallergens may also change over time. This has recently been demonstrated in the Tucson birth cohort study, where the remittance (disappearance) of sensitisation to individual inhalant allergens between the ages of 6 and 11 years was found in up to $20-30 \%$ of children (figure 2). For example, $20 \%$ of children sensitised to grass pollen at the age of 6 were no longer sensitised at age 11 . Overall, $8.2 \%$ of all children in this cohort who had been sensitised to one or more allergens at

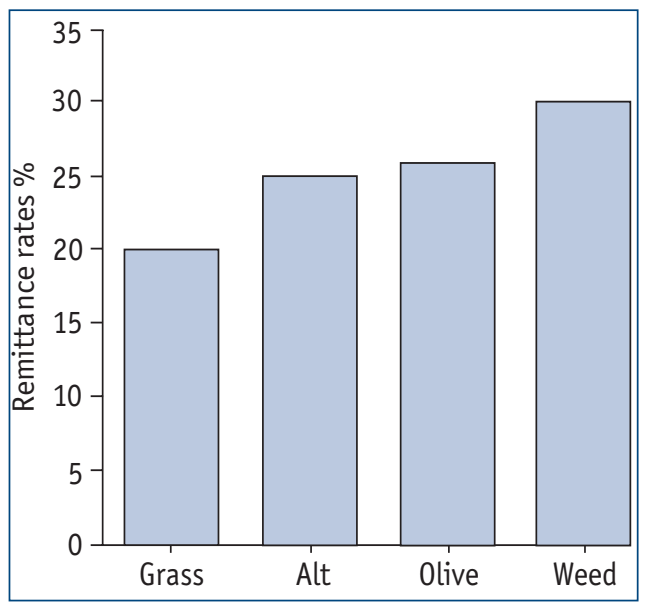

the age of 6 years showed complete disappearance of sensitisation at the age of 11 . This shows that atopic sensitisation to inhalant allergens may disappear during childhood.

\section{Size matters}

Although it is commonly believed that allergic sensitisation is a black-or-white phenomenon, and that it is sensitisation per se that determines the risk of symptoms (you are either allergic or nonallergic to certain allergens), recent studies have shown that the degree of sensitisation is also important. To quote a phrase, "size matters"! For example, in the Manchester birth cohort study [2], the probability of wheeze increased fourfold in children when the degree of sensitisation to cat increased from slightly positive (specific lgE 0.3 $\mathrm{kU}$ per L) to strongly positive (specific lgE $300 \mathrm{kU}$ per L). Similar dose-response relationships were seen for cat and dust mite allergens, and this relationship was even more pronounced when the cumulative degree of sensitisation to the three allergens was calculated. Similar results were recently found in the Swedish BAMSE birth cohort study [3], not only for wheeze, but also for atopic eczema and allergic rhinitis. It appears, therefore, that the degree of sensitisation is at least as important in determining the risk of allergic disease as is allergic sensitisation per se. Similar dose-response relationships between specific IgE levels and symptoms have been found for food allergens. Although these results have been used to suggest that measurements of specific lgE could be helpful in predicting clinical responses to food, thus reducing the use of food challenges, there are two reasons why this may not be true. Firstly, a good predictive value $(>90 \%)$ is only encountered with very high specific IgE levels (>50 kU per L), which are

\section{Figure 2}

Remittance rates between the ages of 6 and 11 years for some common allergens. Alt: Alternaria. Reproduced from [1], with permission from the publisher. 


\section{Educational questions}

Are the following statements true or false?

1. Total IgE measurement is not a useful procedure in the diagnosis of allergy.

2. A positive skin test or specific IgE test to an allergen confirms allergy to that allergen.

3. Although sensitisation to foods is commonly transient, sensitisation to inhalant allergens is likely to persist in almost all children for many years.

4. The results of studies from (inter)national referral centres are likely to change the way I am working in my practice. encountered very infrequently in clinical practice. Secondly, even with these high specific IgE levels, the prediction of a positive response never reaches $100 \%$. In other words, it is never possible to predict the clinical response to food reliably in every child based on their food-specific IgE levels. In truth, a food challenge is the only way to reliably diagnose food allergy in children.

\section{Food challenges}

The diagnosis of food allergy is based on elimination and challenge. Symptoms should improve when the suspected food is removed from the diet. If symptoms don't improve, food allergy is excluded, and the food is reintroduced. If symptoms do improve, a challenge follows. The basic rule is that if symptoms reappear with challenge, the diagnosis of food allergy can be made. Unfortunately, open challenge procedures have been shown to carry a high risk of false-positive results, predominantly if the index of suspicion of food allergy is high in parents, physicians or both. For this reason, the gold standard of food allergy diagnosis is the double-blind placebocontrolled food challenge (DBPCFC). For example, in a series of DBPCFCs carried out by the author and colleagues, two-thirds of all diagnoses of cow's milk allergy in infants and children (based on open elimination and challenge) were excluded with DBPCFCs.

Although DBPCFCs are relatively complex time- and labourintensive procedures, they are not difficult to perform once you have the appropriate recipes. Various validated challenges have been published and can be found in the reference list. The use of natural foods to hide the suspect food is recommended, over the use of capsules, because children are more likely to eat natural foods. For example, egg can be hidden in pancakes, or peanuts hidden in cookies, which children like to eat.

It is commonly stated that DBPCFCs are not needed in children with severe or objective symptoms. The author and others, however, have found skin rashes, flare-ups of eczema and vomiting on placebo days in many cases. Similarly, it has been suggested that DBPCFCs are not needed in infants because infants will not fake symptoms. True as this may be, the author and others encountered symptoms on up to $25 \%$ of placebo challenges in infants and young children. These would have been incorrectly diagnosed as positive responses in open challenges. Therefore, it seems reasonable to conclude that
DBPCFCs are really the only way to reliably diagnose or exclude food allergy in children, and that the effort involved is worthwhile. With the published experience of others, it should be possible for physicians to go out and just do it!

Finally, a word of caution about interpreting the results of studies from leading centres. Please take a moment to realise that the population of patients in such centres is most likely to differ quite dramatically from the patients in your centre. Although it is important in all studies to consider whether the patients in the particular study are comparable to the ones you are treating in your practice, this may be particularly critical in studies coming from top-ofthe-bill (inter)national referral centres. For example, in a study on the role of food allergy in atopic eczema, results from DBPCFCs in a national German referral centre showed that food allergy was found in more than $50 \%$ of patients with eczema. In our own series in a large district general hospital, only 10\% of patients with eczema had a positive DBPCFC, $66 \%$ of whom did not respond to the offending food with increased eczema, but with other symptoms (urticaria, erythema, vomiting). Another example comes from a national referral centre for food allergy in the USA, where respiratory symptoms were found in 17\% of DBPCFCs; in our own hospital, it is $<2 \%$. Interestingly, the US study reported IgE levels in its population of children and young adults. The median total IgE level in this group was 3,400 kU per L, showing that this was a highly selected group of extremely atopic individuals. Again, size matters, as the results of studies in such an extreme population should not be used as a basis for decisions that are intended for use in more average populations.

\section{Conclusion}

Allergy tests don't really exist (unless you mean challenge tests), but sensitisation tests do. SPTs, because of their low cost and rapid and visual availability, are the best tools for screening for allergy. Specific IgE tests, because they are more costly, might better be reserved for confirming or excluding the suspicion of specific sensitisations, and may be used for sensitisation screening in patients using antihistamines or those with severe eczema. Size matters, at least in allergy diagnosis. It is not only sensitisation per se, but also the degree of sensitisation, that determines the risk of allergic disease. If you really want to know whether a food is causing symptoms, test it by DBPCFC. 


\section{References}

1. Stern DA, Lohman IC, Wright AL, Taussig LM, Martinez FD, Halonen M. Dynamic changes in sensitization to specific aeroallergens in children raised in a desert environment. Clin Exp Allergy 2004; 34: 1563-1669.

Fascinating paper showing quite pronounced changes over time in the degree of sensitisation to inhalant allergens in children aged 5-10 yrs.

2. Simpson A, Soderstrom L, Ahlstedt S, Murray CS, Woodcock A, Custovic A. IgE antibody quantification and the probability of wheeze in preschool children. J Allergy Clin Immunol 2005; 116: 744-749.

Size matters! It is not only sensitisation per se, but also the degree of sensitisation that determines the risk of symptoms.

3. Wickman M, Ahlstedt S, Lilja G, Hamsten MH. Quantification of IgE antibodies simplifies the classification of allergic diseases in 4-year-old children. A report from the prospective birth cohort study-BAMSE. Pediatr Allergy Immunol 2003; 14: 441-447. The same message: size matters.

\section{Suggested answers}

1. True

2. False

3. False

4. True, but not in a direct

way

\section{Further reading (key references indicated by*) \\ Review articles}

* Host A, Andrae S, Charkin S, et al. Allergy testing in children: why, who, when and how? Allergy 2003; 58: 559-569.

This is a review of allergy diagnostic techniques, including skin tests and specific IgE tests. Written by a large group of European experts. Covers the basics of allergy testing.

Frew AJ. Skin tests in clinical practice and epidemiology. Clin Exp Allergy 1992; 22: 881-882.

Old review paper, but a very clear and concise overview of skin tests.

Heinzerling L, Frew AJ, Bindslev-Jensen C, et al. Standard prick testing and sensitization to inhalant allergens across Europe: a survey from the GA2LEN network. Allergy 2005; 60: 1287-1300.

This is a comprehensive review of the choice of allergens for skin-prick testing in Europe. Insightful when you have to consider how to test a child who has moved in from abroad.

\section{Skin testing: results from individual studies}

Vasar M, Julge K, Björkstén B. Development of atopic sensitization and allergic diseases in early childhood. Acta Paediatr 2000; 89: 523-527.

\section{Skin test data in infants and toddlers}

Arshad SH, Tariq SM, Matthews S, Hakim E. Sensitization to common allergens and its association with allergic disorders at age 4 years: a whole population birth cohort study. Pediatrics 2001; 108: E33.

Provides results of skin prick testing in a UK birth cohort at age 4 years.

Roberts G, Peckitt C, Northstone K, et al. Relationship between aeroallergen and food allergen sensitization in childhood. Clin Exp Allergy 2005; 35: 933-940.

Provides results of skin prick testing in a very large UK birth cohort at age 7 years.

\section{Specific IgE testing: results from individual studies}

Kulig M, Bergmann R, Klettke U, Wahn V, Tacke U, Wahn U. Natural course of sensitization to food and inhalant allergens during the first 6 years of life.

J Allergy Clin Immunol 1999; 103: 1173-1179.

Changes in specific IgE sensitisation rates from birth to the age of 6 years in a German birth cohort.

\section{Food challenges}

Kaila M, Isolauri E. Diagnosis of cow's milk allergy: open or blinded? J Allergy Clin Immunol 1997; 100: 714-715.

Paper showing that open challenges carry a much larger risk than double-blind placebo-controlled challenges of false-positive results.

Sampson HA. 9. Food allergy. J Allergy Clin Immunol 2003; 111: Suppl. 2, S540-S547.

Review paper on food allergy and DBPCFCs, written by one of the leading experts in the field. It is important to realise that the results obtained in a setting such as Professor Sampson's are from highly selected patient populations, which may not be directly applicable in other cohorts of patients.

Vlieg-Boerstra BJ, Bïleveld CM, van der Heide S, et al. Development and validation of challenge materials for double-blind, placebo-controlled food challenges in children. J Allergy Clin Immunol 2004; 113: 341-346.

Superb paper showing the difficulties in obtaining good-quality blinded recipes for DBPCFC. The authors are kind enough to share their validated recipes in an online data repository. Data to be read and used! 